Journal of Computational Acoustics, Vol. 25, No. 1 (2017) 1630001 (19 pages)

(C) The Author(s)

DOI: $10.1142 / S 0218396 X 16300012$

\title{
Review: The Use of Equivalent Source Method in Computational Acoustics
}

\author{
Seongkyu Lee \\ Mechanical and Aerospace Engineering \\ University of California, Davis \\ Davis, CA 95616, USA \\ skulee@ucdavis.edu
}

Received 19 April 2015

Accepted 25 July 2016

Published 11 November 2016

\begin{abstract}
This paper reviews the equivalent source method (ESM), an attractive alternative to the standard boundary element method (BEM). The ESM has been developed under different names: method of fundamental solutions, wave superposition method, equivalent source method, etc. However, regardless of the method name, the basic concept is very similar; that is to use auxiliary points called equivalent sources to reconstruct the acoustic pressure for radiation or scattering problems. The strength of the equivalent sources are then determined via various approaches such that the boundary conditions on the boundary surface are satisfied. This paper reviews several frequencydomain and time-domain ESMs. There are several distinct advantages in these types of methods: (1) the method is a meshless approach so that it is easy and simple to implement; (2) it does not have a numerical singularity problem that occurs in the BEM; (3) the number of equivalent sources can be fewer than the number of surface collocation points so that the matrix size is reduced and a fast computation is achieved for large problems. The main issue of the ESM is that there is no rule to find out the optimal number and position of equivalent sources. In addition, the ESM suffers from the numerical instability that is associated with the ill-conditioned matrix. Some guidelines have been suggested in terms of finding the number and position of the sources, and several numerical techniques have been developed to resolve the numerical instability. This paper reviews the common theories, numerical issues and challenges of the ESM, and it summarizes recent developments and applications of the ESM to aircraft noise.
\end{abstract}

Keywords: Equivalent source method; method of fundamental solutions; wave superposition method; boundary element method; acoustic radiation; acoustic scattering, aeroacoustics.

\section{Introduction}

Acoustic radiation and scattering are fundamental problems in computational acoustics. The boundary element method (BEM) ${ }^{1,2}$ has been widely used to solve these problems for several decades. One advantage of the BEM over finite difference or finite element methods

\footnotetext{
This is an Open Access article published by World Scientific Publishing Company. It is distributed under the terms of the Creative Commons Attribution 4.0 (CC-BY) License. Further distribution of this work is permitted, provided the original work is properly cited.
} 


\section{S. Lee}

is that it does not require the entire domain solution since it requires the discretization only on the boundary of the domain. The second advantage is that it does not involve numerical dissipation and dispersion errors since the free-space Green's function provides the direct relation of the pressure at a receiver from a source. The BEM requires mesh on the surface where the numerical integration is carried out. Often, quadrature or triangular meshes are used. When the boundary integral equation is solved on the surface, the self-mesh computation results in a singularity problem as the distance between the source and the surface point (collocation point) becomes zero. This singularity problem should be carefully resolved using additional numerical techniques. ${ }^{3-6}$

An alternative method to the BEM has been developed and applied for acoustic radiation and scattering problems. The method uses equivalent sources that are located outside the acoustic domain, i.e. inside for an exterior problem or outside for an interior problem. The equivalent sources generate relevant or approximate sound fields. A schematic of this method is shown in Fig. 1 for an exterior problem. There are several advantages in this method over the traditional BEM. First, this method is meshless since it uses simple point sources inside or outside the surface. The meshless method allows to avoid the discretization of the surface and the associated numerical integration around the mesh, simplifying the numerical algorithm and significantly reducing the coding effort. The second advantage is that it does not involve the numerical singularity issue since the distance between the collocation point and the equivalent source point is not equal to zero. This advantage also allows to avoid difficult numerical integrations to handle the singularity problem. The third advantage is that the number of equivalent sources is not necessarily the same as the number of collocation points. The number of equivalent sources is a control variable, and the accuracy and the computation time can be exchanged with this control variable. A smaller number of equivalent sources can significantly reduce the size of the linear system and the computation time.

There are disadvantages in this method too. The solution may not be as accurate as that of the BEM. This method only provides approximate solutions with fewer equivalent sources than the surface collocation points. However, the error is only within a few percentages with

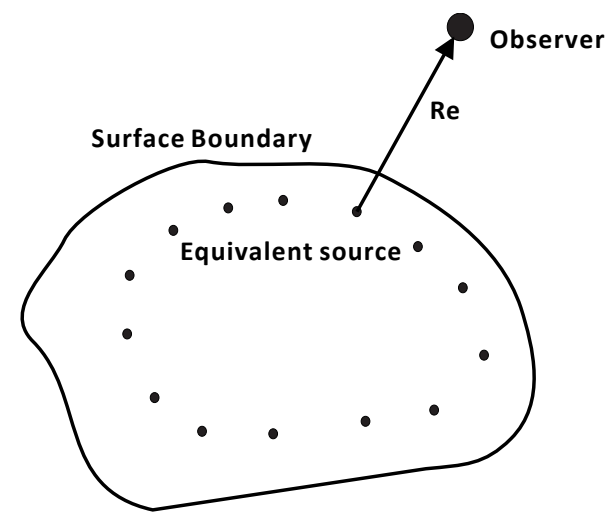

Fig. 1. Equivalent sources embedded inside the radiating or scattering surface. 
a reasonable number of equivalent sources, making the approximate solution acceptable for most engineering problems. Another and a more serious issue is that the method often encounters the numerical instability problem since the linear algebraic system becomes illconditioned. Regularization techniques or robust numerical algorithms are typically needed to resolve this instability issue.

This alternative method has been used under different names by different groups. For example, the method is called the method of fundamental solutions, wave superposition method, equivalent source method (ESM), source simulation technique, multipole method, Trefftz method, etc. However, the fundamental theory and numerical algorithm remain very similar among these approaches. Different research groups prefer to give their method alternative names or simply did not recognize similar work in different fields. This paper uncovers and connects the common grounds, issues, challenges, and improvements across these methods.

Ochmann ${ }^{7}$ presented an excellent summary of these methods for acoustic radiation problems from a theoretical point of view. However, this paper was published 20 years ago, and it is worthwhile to review recent developments in this method for both acoustic radiation and scattering problems. Additionally, the method of fundamental solutions was not covered in Ochmann's review paper.

The goal of this review paper is to collect all the relevant literatures in the essentially same area as the alternative BEM method and to summarize the progress and development of the method over recent decades. This paper chooses the name of ESM to describe the alternative method since it is more widely accepted and has been used in the engineering acoustic community recently and it properly reflects the principle of the method. However, the current paper covers literatures that were published under the name of the method of fundamental solutions, wave superposition method, and ESM.

\section{Method of Fundamental Solutions}

The method of fundamental solutions (MFS) is applied to the numerical solution of elliptic boundary value problems. While the wave superposition method or the ESM that will be discussed in the next sections have been mainly developed for acoustic or elastodynamic problems, the MFS is a more general approach that was applied to various elliptic partial differential equations in two and three space variables including potential, Helmholtz, and diffusion problems. The MFS has been mainly developed in the context of mathematics rather than engineering problems. Therefore, numerical algorithms and rigorous theoretical proofs have been the main focuses in the literature.

In the MFS, the approximate solution is found in the form of a linear superposition of source functions or fundamental solutions. The idea was originally published by Kupradze and Aleksidze. ${ }^{8}$ The MFS has been applied to the potential flow, ${ }^{9}$ the biharmonic equation, ${ }^{10}$ the Poisson equation, ${ }^{11}$ the Stokes flow, ${ }^{12,13}$ and the diffusion equation. ${ }^{14,15}$ The first application of the MFS to acoustic problems was presented by Kondapalli et al. ${ }^{16}$ A review of this method was given in several of the literatures. ${ }^{17-19}$ The current paper will focus on the application of the MFS to acoustic problems. 
Let us consider an acoustic radiation problem with the Helmholtz equation

$$
\left(\nabla^{2}+k^{2}\right) p=0 \quad \text { in } \Omega,
$$

where $\nabla$ is the Laplacian, $k$ is the wavenumber, $\Omega$ is the fluid domain, and $p$ is the acoustic pressure. On the surface, the boundary condition is given as

$$
\frac{\partial p}{\partial n_{\beta}}=f,
$$

where $\beta$ indicates an arbitrary position on the surface that is called the collocation point and $n_{\beta}$ is the unit normal vector on the collocation point. A variable of $f$ is a given function to describe the boundary condition. The infinite condition is the Sommerfeld radiation condition.

$$
\lim _{r \rightarrow \infty} r\left(\frac{\partial p}{\partial r}-i k p\right)=0,
$$

where $r$ is the distance between the source and receiver.

A fundamental solution of the Helmholtz equation (1) is

$$
p_{f}=A \exp (-i k r) / r,
$$

where $A$ is a complex constant. The MFS typically uses equivalent sources outside the surface. The superposition of this fundamental solution for $N$ points approximates the solution of Eq. (1)

$$
\tilde{p}=\sum_{\alpha=1}^{N} A_{\alpha} \frac{\exp \left(-i k r_{\alpha}\right)}{r_{\alpha}},
$$

where $\tilde{p}$ is the approximate solution and the subscript $\alpha$ is the index of the source.

Then, the boundary condition (2) at an arbitrary point on the surface $(\partial \Omega)$ becomes

$$
\frac{\partial \tilde{p}}{\partial n_{\beta}}=-\sum_{\alpha=1}^{N} A_{\alpha} \frac{r_{\alpha \beta}}{\partial n_{\beta}} \frac{\exp \left(-i k r_{\alpha \beta}\right)}{r_{\alpha \beta}^{2}}\left(i k r_{\alpha \beta}+1\right)=f_{\beta},
$$

where $r_{\alpha \beta}$ denotes the distance between the $\alpha$ th equivalent source and $\beta$ th collocation point. As discussed earlier, $r_{\alpha \beta}$ is not zero for all points since the source position is not the same as the collocation point so that the singularity problem does not occur in this method.

If the total number of collocation points is $M$, Eq. (6) provides a linear algebraic system of equations or a matrix with a size of $M \times N$ for a fixed position of the equivalent sources.

$$
[C][A]=[F],
$$

where $[C]$ denotes the coefficient matrix, $[A]$ denotes a vector of the equivalent source strength, and $[F]$ is a vector of the boundary condition on the surface.

Note that there is no need that $N$ is equal to $M$. The approximate solution can be obtained with a fewer number of $N$ than $M$. The smaller size of the matrix can reduce 
the computation time significantly, which is an advantage of this method. In this case, the least-square problem can be formulated.

$$
S=\sum_{\beta=1}^{M}\left|g_{\beta}\right|^{2}
$$

where

$$
g_{\beta}=f_{\beta}+\sum_{\alpha=1}^{N} A_{\alpha} \frac{r_{\alpha \beta}}{\partial n_{\beta}} \frac{\exp \left(-i k r_{\alpha \beta}\right)}{r_{\alpha \beta}^{2}}\left(i k r_{\alpha \beta}+1\right) .
$$

We seek to minimize the least-squares function, Eq. (8). There are many least-square solvers. The application of the least-square solvers to the MFS can be found in the literatures. ${ }^{10,20-22}$ Smyrlis defined appropriate discrete Sobolev norms and developed a weighted least-squares MFS algorithm. ${ }^{23}$

The MFS was also applied to acoustic scattering problems. ${ }^{24-30}$ For an acoustic scattering problem, the total acoustic pressure is divided into the incident and scattered pressure.

$$
p=p^{i}+p^{s},
$$

where $p^{i}$ is the incident pressure and $p^{s}$ is the scattered pressure. Typically, the incident pressure field is given. Equation (10) provides the Helmholtz equation for the scattered pressure

$$
\nabla^{2} p^{s}+k^{2} p^{s}=0 \quad \text { in } \Omega
$$

and the boundary condition on the rigid scattering surface gives

$$
\frac{\partial p^{i}}{\partial n_{\beta}}+\frac{\partial p^{s}}{\partial n_{\beta}}=0
$$

In this case, the least-square problem becomes

$$
g_{\beta}=\frac{\partial p^{i}}{\partial n_{\beta}}-\sum_{\alpha=1}^{N} A_{\alpha} \frac{r_{\alpha \beta}}{\partial n_{\beta}} \frac{\exp \left(-i k r_{\alpha \beta}\right)}{r_{\alpha \beta}^{2}}\left(i k r_{\alpha \beta}+1\right) .
$$

The position of equivalent sources does not need to be fixed. It can be treated as unknowns and determined in the solution process. Mathon and Johnston ${ }^{31}$ first used moving or floating sources for the MFS. They used the locations of the sources as parameters to be determined by the algorithm along with the magnitude of the sources. This increases the number of unknowns from $N$ to $4 N$ due to the Cartesian coordinates of the $N$ sources. The resulting equations are the nonlinear least-square equations that can be solved by standard codes. Kondapalli et al. ${ }^{16}$ also used moving sources for acoustic wave scattering in fluids. They discussed how the moving sources achieve the same level of accuracy with the fewer source points. The moving source concept also removes the difficulty of finding the optimal positions of the source points. However, this problem significantly increases the computation 
time. The moving source concept is also used in the inverse acoustic scattering problem, ${ }^{32}$ where the word moving should not be understood as the motion of a body.

Much of the literature addressed the stability issue that is associated with the position of equivalent sources. Kitagawa ${ }^{33,34}$ first found the numerical stability issue in the MFS. The stability and convergence issue was discussed in the literature. ${ }^{35}$ Drombosky et al. ${ }^{36}$ showed that the effective condition number provides an estimate of the quality of the MFS solution. Bogomolny ${ }^{18}$ and Kondapalli et al. ${ }^{16}$ pointed out that the ill-conditioning is not associated with internal resonance critical frequencies. Rather, it is due to the nature of the method. A singular value decomposition (SVD) method was suggested to regularize the ill-conditioned matrix. ${ }^{37-39}$ Lin et al. ${ }^{40}$ and Wei et al. ${ }^{39}$ investigated different regularization techniques for the MFS using Tikhonov regularization and damped SVD strategy.

Some interesting applications of the MFS are the boundary singularity problem, ${ }^{41}$ the eigenvalue problem, ${ }^{42}$ room acoustics, ${ }^{43,44}$ and underwater acoustics. ${ }^{45,46}$

\section{Wave Superposition Method}

A very similar method to the MFS was studied and published under the name the wave superposition method (WSM). The main difference between the MFS and the WSM is that the MFS uses randomly distributed sources and the WSM uses an auxiliary surface to locate the sources. Further, the MFS uses fewer equivalent sources than the collocation points so that the resulting under-determined matrix can be solved using a least-square algorithm. The earlier WSM uses the same number of sources as the collocation points.

Since many independent numerical studies have been studied using the WSM, it is useful to review the literatures published under this category. Koopmann et al. ${ }^{47}$ first proposed the idea of the WSM. Their idea was that the combined fields of an array of sources interior to a radiator can be made to reproduce a velocity prescribed on the surface of the radiator. The WSM determines the source strength (unknown) given the velocity boundary condition (known) using the derivative of the free-space Green's function (kernal) as shown in

$$
u(\mathbf{r})=\int_{\mathbf{V}} q\left(\mathbf{r}_{0}\right) \nabla G\left(\left|\mathbf{r}-\mathbf{r}_{0}\right|\right) d \mathbf{V}\left(\mathbf{r}_{0}\right),
$$

where $u$ is the velocity on the surface, $V$ is the domain of equivalent sources, $q$ is the source strength, $G$ is the free-space Green's function, $\mathbf{r}$ is the observer coordinate, and $\mathbf{r}_{0}$ is the equivalent source coordinate. Koopmann et $a .^{47}$ proved that the superposition integral is equivalent to the Helmholtz integral. It is apparent that they did not recognize the earlier similar work — the MFS. However, subsequent publications from this group and others have independently contributed to the advancement of this method.

Earlier work addressed that the ESM is more accurate and more efficient than the BEM. Miller et $a .^{48}$ compared the ESM (or WSM) with the BEM for acoustic scattering for rigid bodies or elastic shell structures. Figure 2 shows a comparison between the BEM and the WSM for the scattering by a cylindrical shell. They mentioned that the ESM does not appear to be affected by spurious internal resonances so that it is more accurate than the 


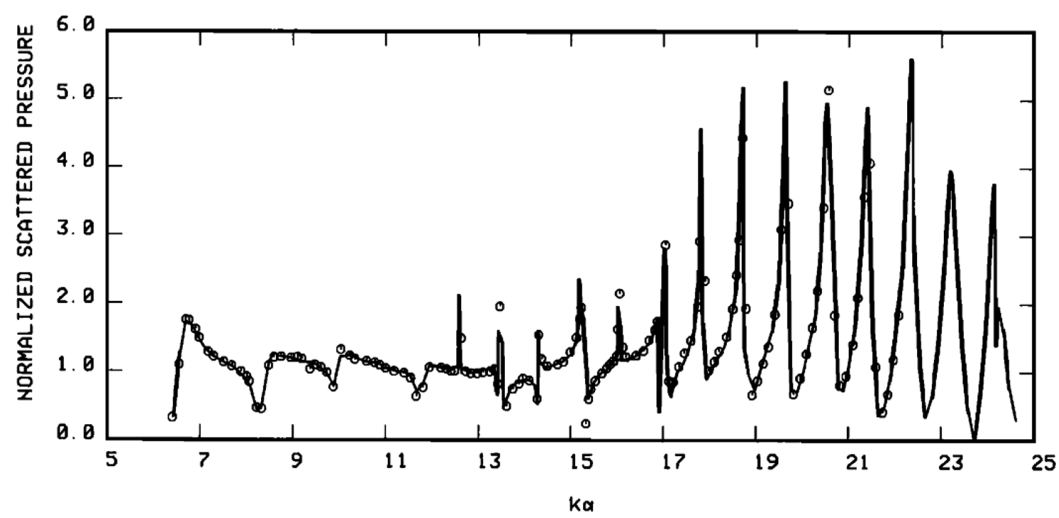

Fig. 2. Normalized backscattered form function from a cylindrical shell with hemispherical endcaps for endon incidence for BEM/CHIEF (circles) and WSM (solid). ${ }^{48}$ ( $k$ is a wavenumber and $a$ is the radius of the cylinder.)

BEM, especially at high frequency. They mentioned that the equivalent positions that are located at the half minimum radius of the body from the surface provide excellent results. Burgess and Mahajerin ${ }^{49}$ compared the ESM with the BEM for plane elastic problems. They mentioned that the implementation of the ESM is much easier than the BEM since the ESM does not involve surface integrations. They showed that the BEM has the strong diagonal in the influence matrix and the ESM has the weaker diagonal. Therefore, the matrix becomes ill-conditioned in the ESM. However, they addressed that this ill-conditioned matrix does not cause a significant issue with determining the accuracy of the solution. They discussed that the position of the equivalent sources can be a concern, but they found that the results differ only by a few percent. These earlier work about the comparison between the ESM and the BEM should be carefully treated. Their conclusions could mislead readers since they do not capture full capabilities and limitations of the ESM. The numerical issues and the limitations of the ESM such as the sensitivity of the solution to the number and position of the equivalent sources as well as the numerical instability were not fully investigated or understood at that time. These issues were addressed and studied in more detail later.

Jeans and Miller ${ }^{50}$ compared single and double layer sources and point sources and integrated surfaces. They discussed the numerical stability issue associated with the position of equivalent sources. They suggested that the source nodal points need to be placed at a fixed distance along the normal direction from the boundary nodal points. Song et al. ${ }^{51}$ studied numerical errors in the WSM. They introduced velocity interpolation functions to quantify the wave reconstruction errors.

Koopmann et al. ${ }^{47}$ and Miller et al. ${ }^{48}$ presented that the WSM avoids nonuniqueness at critical interior resonance frequencies that occur in the BEM, as discussed earlier. However, the resolution of the nonuniqueness problem in the WSM was questioned by other researchers. For example Wilton et al..$^{52}$ and Leblanc et al..$^{53}$ presented that the WSM is not free of the nonuniqueness problem and they showed that the nonuniqueness problem still exists at critical frequencies. Ochmann ${ }^{7}$ also discussed that the nonuniqueness problem 
occurs in the WSM if the inner surface is closed. He discussed that if the layer method or the multi-point method is used, the nonuniqueness problem can be avoided since the equivalent sources do not enclose an interior space. This can be done by locating equivalent sources along a line or by choosing a surface which is not closed.

Equation (14) appears as a Fredholm integral equation of the first kind. The Fredholm integral equation of the first kind is given as

$$
f(x)=\int_{a}^{b} K(x, t) u(t) d t
$$

where $K$ is a kernel, $u$ is an unknown function and $f$ is a known function. The limits of the integration are fixed. The Fredholm integral equation of the first kind is an ill-posed problem. Fahnline and Koopmann ${ }^{54}$ used the SVD to deal with an ill-posed matrix problem. Small singular values contribute to the numerical instability. However, truncating too many singular values could result in inaccurate solutions.

The WSM has also been applied to inverse reconstruction problems to find real noise sources or the geometry of radiators. ${ }^{55-57}$

\section{Equivalent Source Method}

The $(E S M)$ can be considered an extension of the WSM. In principle, the method is the same as the MFS or the WSM. The strength of the sources is determined such that the boundary condition on the surface is fulfilled. This method utilizes features that were used in both the MFS and the WSM. The equivalent sources are typically located inside the surface and the position of the sources is fixed as in the WSM. The number of equivalent sources is typically smaller than the number of collocation points so that the under-determined matrix should be solved using the least-square method or the SVD as in the MFS.

While the name of the MFS is used more often in mathematics, the ESM is used more widely in the engineering acoustic community. There has been significant development and progress in the ESM in recent decades. Researchers have applied the ESM to realistic application problems with complex geometries such as an aircraft body with the help of the significant increase in computational capability in terms of speed and memory.

Johnson et al. ${ }^{58}$ used the ESM to calculate the sound field inside an enclosure containing scattering bodies. Bouchet et al. ${ }^{59}$ used an equivalent sphere for acoustic radiation along with the SVD. Gounot et al. ${ }^{60}$ compared two variants of the ESM in terms of numerical instability: least-square minimization (LSM) and full-field equations (FFE). ${ }^{61}$ They found that the LSM provides a better solution.

Holste $^{62}$ used the ESM to calculate aircraft engine noise. He used the Lorentz transformation to introduce the mean flow effect in the ESM and also considered nonuniform flow effects by dividing the flow region into the engine duct and freestream areas. Piscoya et al. ${ }^{63}$ used the ESM for the prediction of the sound radiation of flames and they compared the ESM with the BEM and showed good agreement between the ESM and the BEM. Dunn and Tinetti $^{64-72}$ developed a new frequency-domain ESM, named Fast Scattering Code (FSC), 
and applied the FSC into practical aerospace engineering problems such as the scattering of engine noise by an aircraft fuselage. The application of the FSC into acoustic scattering by Hybrid Wing Body (HWB) are shown in Figs. 3 and 4. Dunn and Tinetti took into a consideration of the effects of uniform and nonuniform flows on the scattering. ${ }^{64,65}$ They also implemented the fast multipole $\operatorname{method}^{68}$ and the OpenMP and MPI parallelization ${ }^{70}$ into the ESM to substantially reduce computation time and memory usage and solve highfrequency noise for a large-scale aircraft scattering body. They suggested some guidelines about choosing the number of collocation points, the number and position of equivalent sources. They suggested that the number of collocation points should be approximately $O\left(k_{0}^{2} S_{A}\right)$ where $k_{0}$ is the reference wavenumber and $S_{A}$ is the scattering surface area. The suggested number of equivalent sources is one-third of the number of collocation points. The suggested equivalent source position is a $90 \%$ scaled replica of the scattering body. They did not discuss the numerical stability issue in their papers. The FSC was also applied to rotorcraft acoustic scattering problems. ${ }^{73}$ An absorbing boundary condition, which is important to model aircraft engine liners, was also applied in the frequency-domain ESM. Reimann et al. ${ }^{66}$ applied an absorbing boundary condition in the presence of mean flow to the FSC to study aircraft engine liner optimization for noise reduction. They used Myer's absorbing boundary condition in the presence of flow. ${ }^{74}$

It is important to note that monopole is not the only source type in the ESM. Pavić 75 showed that multipoles perform better than monopoles especially at high frequencies. Ochmann ${ }^{61}$ used spherical wave functions as source functions.

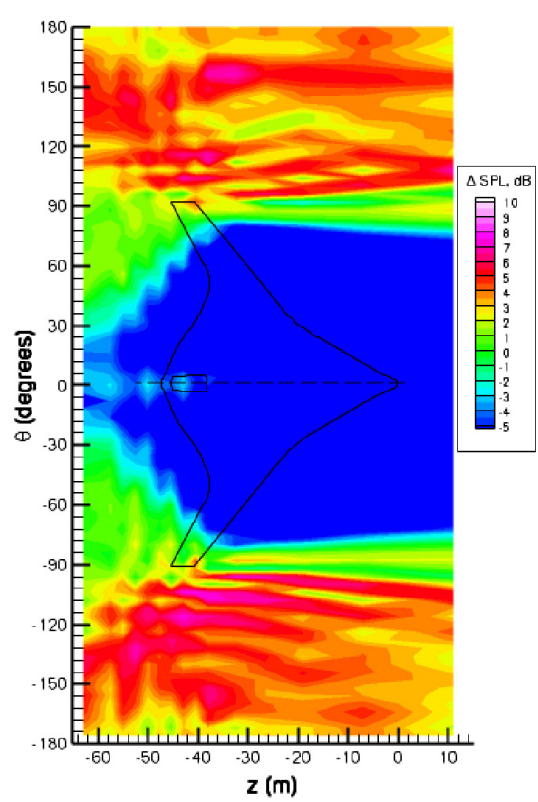

(a) Experimental

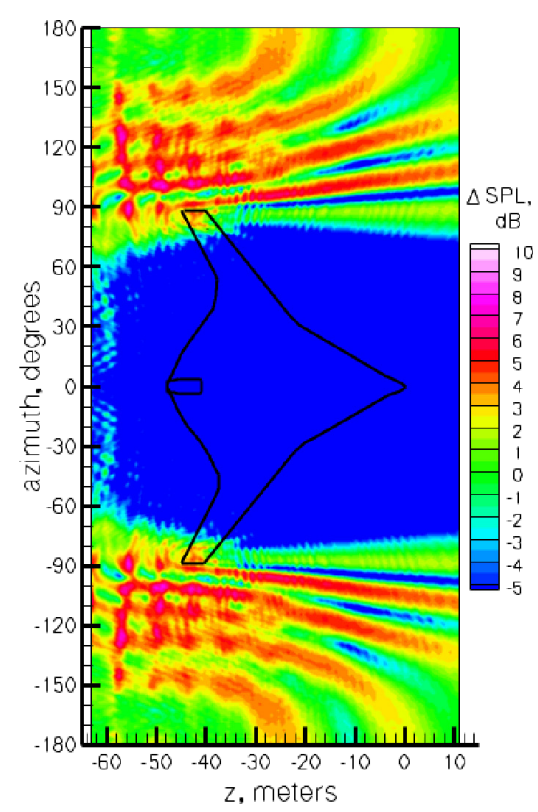

(b) FSC v3.1

Fig. 3. Comparison of the insertion loss contours between measurement and FSC prediction for a frequency of $315 \mathrm{~Hz}$ and a Mach number of 0.0: (a) Experimental data, (b): FSC prediction. ${ }^{69}$ 


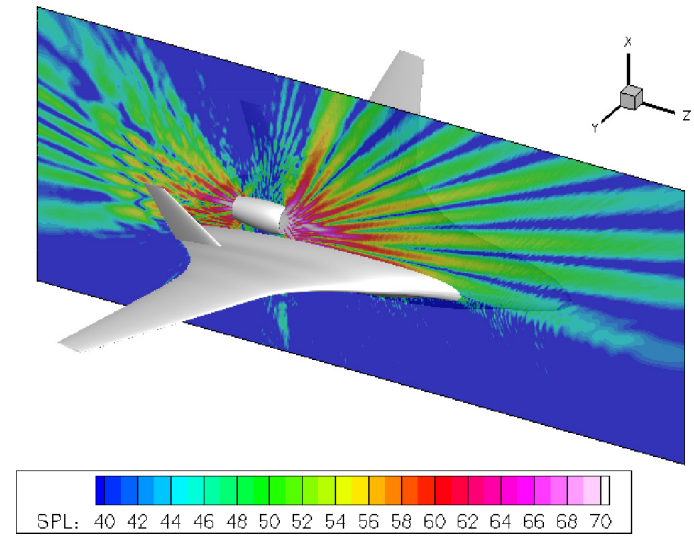

(a) Plane bisecting the starboard nacelle

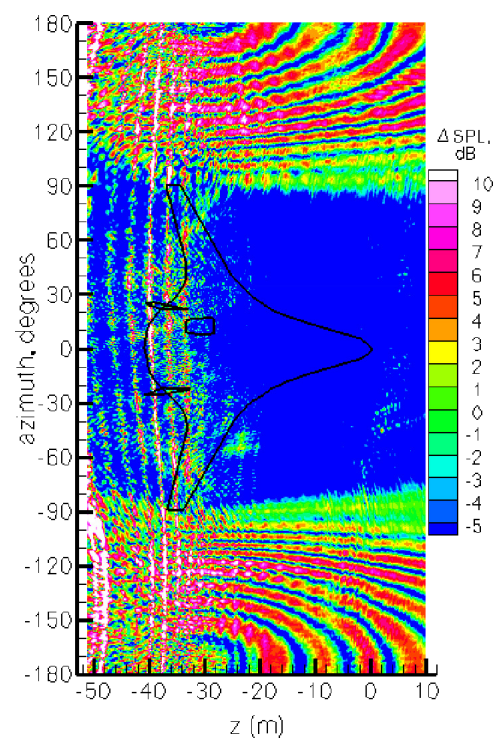

(b) Insertion loss

Fig. 4. Simulations for HWB for a frequency of 1.0 times the fan blade passage frequency and a Mach number of 0.2 (uniform). Incident sound generated by a point source located inside the starboard nacelle at the fan face: (a) Plane bisecting the starboard nacelle, (b) Insertion loss. ${ }^{69}$

Kropp and Svensson ${ }^{76}$ first presented a time-domain ESM for an acoustic radiation problem. This time-domain ESM did not receive much attention and was not been used for practical engineering problems until Lee et al. ${ }^{77-79}$ presented significant improvements of the method and revived it. Lee et al. ${ }^{77-79}$ used analytical formulations of the pressure gradient $^{80}$ for the boundary condition to derive a new formulation to include the effect of a uniform motion of the surface and equivalent sources and to consider the incident field generated by arbitrary moving sources such as rotor blades. They introduced a first-order shape function to discretize the source strength in time and used the SVD to improve the numerical instability issue. ${ }^{77}$ They also introduced a time-averaging algorithm to further remove the numerical instability. ${ }^{78}$

In the time-domain ESM, the strength of equivalent sources is given as a function of time, $q_{e}(t)$. Then the strength of equivalent sources is discretized and expressed using a global shape function

$$
q_{e}(t)=\sum_{l=1}^{\mathrm{NL}} \phi^{l}(t) \tilde{q}_{e}^{l},
$$

where $l$ is the time index and NL is the number of time indices. Here $q_{e}(t)$ is a continuous function with respect to $t$ while $\tilde{q}_{e}^{l}$ is defined discretely at each time-step $l$. Note that the strengths $\tilde{q}_{e}^{l}$ are not known a priori but will eventually be determined. The global shape function, or interpolation function, $\phi^{l}(t)$ plays the role of interpolating the discrete values of $\tilde{q}_{e}^{l}$ onto the function $q_{e}(t)$. A linear global shape function was used in the literatures. ${ }^{77-79}$ 
For rectilinear motion of a slender body or a stationary body with an arbitrary shape, the boundary condition for an acoustically rigid surface can be written as

$$
\frac{\partial p_{s}^{\prime}}{\partial n}=-\frac{\partial p_{i}^{\prime}}{\partial n}
$$

or

$$
\nabla p_{s}^{\prime} \cdot \mathbf{n}=-\nabla p_{i}^{\prime} \cdot \mathbf{n}
$$

where $p_{i}^{\prime}$ and $p_{s}^{\prime}$ are the incident and scattered acoustic pressures, respectively, and $\mathbf{n}$ is the outward unit normal vector on the scattering surface. The right-hand side of Eq. (18) is the incident field, which is known and can be determined by using the analytical formulation for the pressure gradient for arbitrary moving sources. ${ }^{80}$ The left-hand side involves the strength of equivalent sources, which is a main unknown.

The final matrix form is given as follows:

$$
\mathbf{A} \tilde{\mathbf{q}}^{k}=\mathbf{B},
$$

where $\tilde{\mathbf{q}}^{k}$ is a vector form of $\tilde{q}_{e}^{k}$ where $e=1,2, \ldots$, NE. At the $k$ th time-step, the righthand side of Eq. (19) is formed from the incident field and $\tilde{\mathbf{q}}^{l}$, where $l=1, \ldots, k-1$, and the left-hand side gives the contribution at the current time-step. Here the superscript $l$ denotes a running time index and the superscript $k$ a fixed time index of interest, while NL represents the total number of the time index. Before proceeding to the next source time step, the strength of equivalent sources is found. This procedure is repeated as the time step $k$ proceeds from 1 to NL. A and $\mathbf{B}$ can be expressed as

$$
\begin{aligned}
\mathbf{A}(m, e) & =\frac{1}{4 \pi}\left\{\left[\frac{1}{c r_{m e}}\left(\frac{1}{\Delta \tau}\right)+\frac{1}{r_{m e}^{2}} \frac{1}{\Delta \tau}\left(\tau_{m e}-\tau^{k-1}\right)\right]_{\mathrm{ret}}\left[\hat{\mathbf{r}}_{m e}\right]_{\mathrm{ret}} \cdot \mathbf{n}_{m}\right\} \\
\mathbf{B}(m) & =\frac{\partial p_{i, m}^{\prime}}{\partial n}+\frac{1}{4 \pi} \sum_{e=1}^{\mathrm{NE}} \sum_{l=1}^{k-1}\left[\frac{1}{c r_{m e}} \frac{d \phi^{l}\left(\tau_{m e}\right)}{d \tau} \tilde{q}_{e}^{l}+\frac{1}{r_{m e}^{2}} \phi^{l}(\tau) \tilde{q}_{e}^{l}\right]_{\mathrm{ret}}\left[\hat{\mathbf{r}}_{m e}\right]_{\mathrm{ret}} \cdot \mathbf{n}_{m},
\end{aligned}
$$

where $m$ is the index of the surface collocation points, $r_{m e}$ is the distance between the $m$ th collocation point and the eth equivalent source, $\Delta \tau$ is the source time-step, and [ ]ret represents a value evaluated at the retarded time.

The matrix A is usually a rectangular matrix because fewer equivalent sources than surface collocation points are generally used. The matrix equation can be solved using the $\mathrm{SVD}^{77-79}$ to find the least-squares solution of the system which is ill-conditioned. Note that truncating too many singular values results in inaccurate solutions. Although Eqs. (20) and (21) hold only for a stationary body, Lee et al. ${ }^{77}$ also provided a general ESM formula for a moving body with a uniform speed.

Lee et $a l^{78}$ investigated the numerical sensitivity of the solution to the parameters including the number of collocation points, the number of equivalent sources, the position of equivalent sources, the time step, the cut-off singular value, etc. and suggested some guidelines. ${ }^{78}$ The suggested number of equivalent sources is a quarter of the collocation 


\section{S. Lee}

points and the suggested position of the sources is between $80 \%$ and $90 \%$ scale of the surface. When the number of equivalent sources is a quarter of the number of the collocation points and the position of equivalent sources is a $80 \%$ scale of the surface, the maximum error was found to be less than $4 \%$ for the scattering of a point source by a sphere. When the number of equivalent sources is a half of the number of the collocation points, the maximum error was reduced to $2 \%$. They have also clearly demonstrated the advantages of the timedomain method over the frequency-domain method in broadband noise and transient noise problems. Figure 5 shows the scattering of broadband noise by a sphere with a radius of $a$. They also applied their method to rotorcraft noise scattering problems. ${ }^{79}$ They found that the method does not suffer from the nonuniqueness problem at critical frequencies, but this should be further investigated. If the frequency-domain method has the nonuniqueness problem, the time-domain should also have the same problem.

Lee et $a l^{78}$ introduced a time-averaging algorithm ${ }^{81-84}$ to further reduce the numerical instability. The use of the SVD allows to remove small singular values that contribute to the numerical singularity. However, stabilizing the solution with the truncation of singular values can also result in inaccurate solutions. The advantage of using the time-averaging scheme is that it helps keep as many singular values as possible or minimize the number of the truncation of singular values to find out the solution without having the numerical instability. In principle, the averaging scheme stabilizes the solution by filtering out the high frequency components. The time-averaging is applied at each time-step by averaging the equivalent source strength for various time-steps,

$$
\bar{q}^{k-1}=\frac{1}{4}\left(\bar{q}^{k-2}+2 q^{k-1}+q^{k}\right)
$$

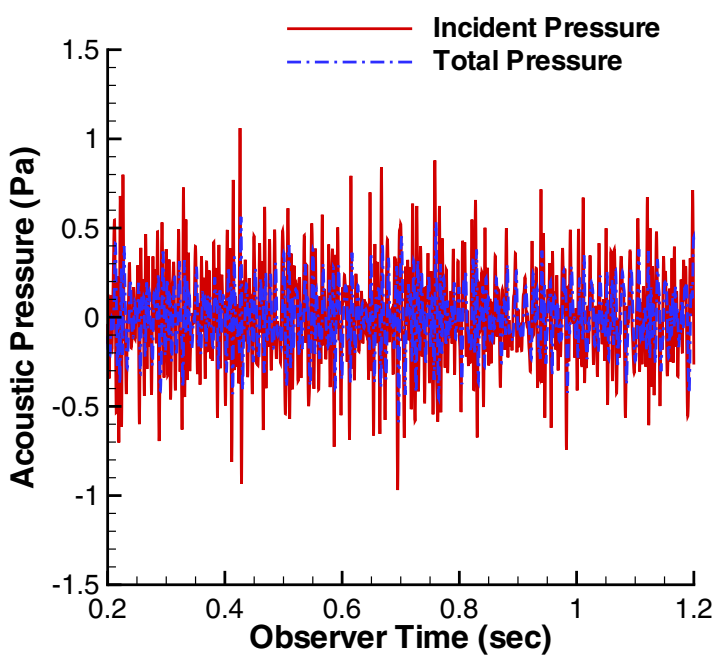

(a)

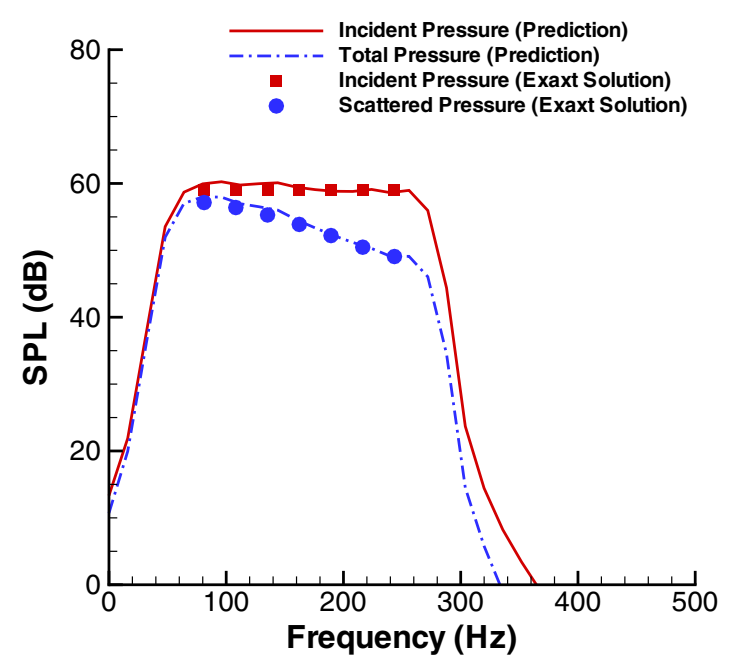

(b)

Fig. 5. Comparison of the incident and total pressure at $r / a=1.2$ and $\theta=154.8 \mathrm{deg}$ : (a) Time history of the pressure, (b) SPL in the frequency domain ${ }^{74}$ ( $r$ is the observer distance, $\theta$ is the observer azimuthal angle.) 


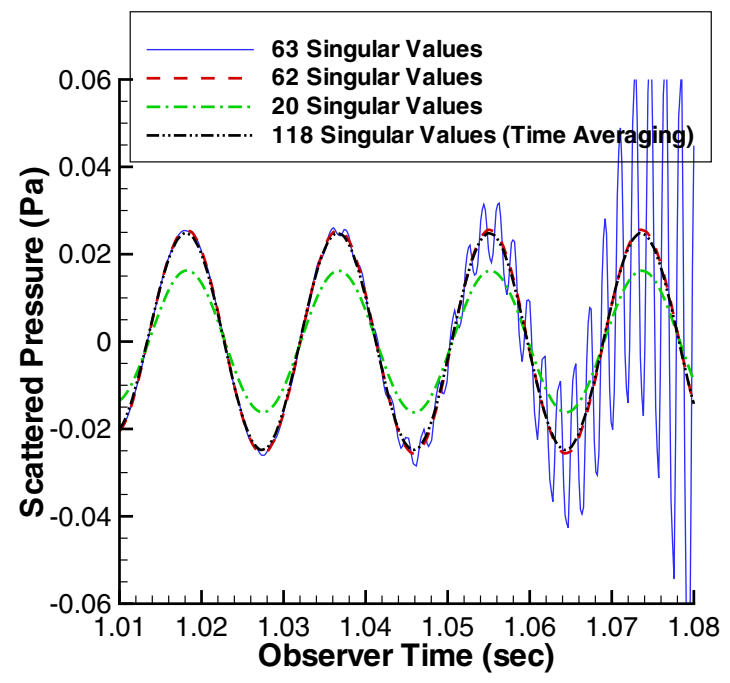

(a)

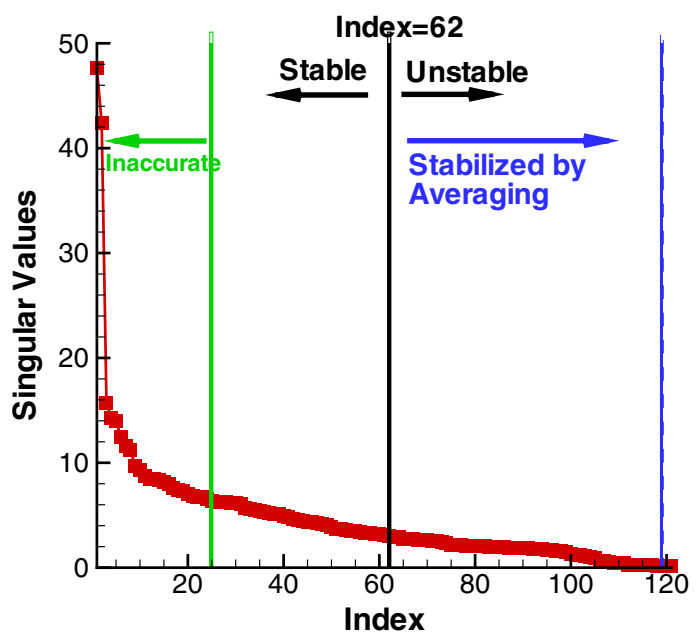

(b)

Fig. 6. Numerical instability with the number of singular values and the resolution of the instability with the time-averaging: (a) Scattered pressure with a different number of singular values used and stabilization of the pressure by the time-averaging scheme for $k a=1$, (b) Singular value distribution and a schematic of the stabilization of the solution by the time-averaging scheme. ${ }^{75}$

where the overbar denotes the averaged quantity and the averaged value is obtained for the previous time-step, $k-1$, while the nonaveraged solution is obtained at the current time-step, $k$. The averaged strength of equivalent sources is used to find the scattered pressure. Figure 6 shows an example of the numerical instability with a different number of singular values and the resolution of the instability with the time averaging scheme. With 63 singular values, the solution exhibits a numerical instability as time goes. By discarding one singular value or using 62 singular value, a stable solution is achieved. If too many singular values are truncated and only 20 singular values are maintained, the solution has a large error even though the instability is avoided. However, if the time averaging is used, the solution is stable even with a large number of singular values, 118 singular values. Therefore, the time averaging provides the most accurate results with a large number of singular values or a smaller number of the truncation of singular values.

Swift et al. ${ }^{85}$ extended this time-domain ESM to solve acoustic scattering by a planar surface. They used the co-location of equivalent sources and collocation points and the same number of equivalent sources with collocation points. Therefore, their method is similar to BEM and it does not carry some advantages of the ESM, but they showed that their method is more accurate than Lee's method for acoustic scattering by a planar surface. Bi et al. ${ }^{86}$ used a cubic spline interpolation in the discretization of the source in time. Recently, the time-domain ESM has been used in sound visualization and near-field acoustical holography. ${ }^{87-93}$

The time-domain ESM was only used for the rigid boundary condition. However, the absorbing boundary condition in the presence of flow in the time domain ${ }^{94}$ is available. 
This boundary condition can be applied to the time-domain ESM. A challenge in the timedomain ESM is to solve high-frequency noise for a large-scale scattering body. Recently, a fast time-domain BEM $^{95}$ was developed using fast multi-level and multi-grid algorithms and Graphics Processing Unit (GPU) computing. This technique can also be applied to the time-domain ESM to solve high-frequency noise for a large-scale scattering body.

\section{Concluding Remarks}

The current paper reviews fundamental theories, numerical issues, and recent improvements of the ESM. The ESM is a meshless method so that it significantly simplifies the implementation of the method and the coding effort. It also uses fewer equivalent sources than the boundary collocation points, providing a faster computation than the BEM. The ESM does not have a numerical singularity problem. For many cases, it does not suffer from the nonuniqueness problem at critical frequencies, although this problem depends on the numerical algorithm and the positioning of equivalent sources.

A critical problem in the ESM is that there is no rule in terms of the number and position of equivalent sources. Gounot and Musafir ${ }^{96}$ used simple geometries to suggest a suitable location and array of equivalent sources. They suggested the nondimensional frequency $k L$ for the suitable number of monopoles. They investigated the effect of the incident wave vector and the aspect ratio of the surface on the single or double array of equivalent sources. Dunn and Tinetti ${ }^{64}$ showed that one-third of the number of collocation points and a $90 \%$ scaled replica of the scattering body provide good results. Lee et al. ${ }^{78}$ provided some guidelines for the number and position of equivalent sources in the time domain. They stated that a quarter of the collocation points and an $80 \%$ to $90 \%$ smaller replica of the scattering body provide good results. Kondapalli et al. ${ }^{16}$ discussed that the accuracy of the solutions tends to increase as the sources are positioned further away from the surface. However this also causes the matrix to become more ill-conditioned. A similar trend was also found in the literature. ${ }^{78}$ Gounot and Musafir used a genetic algorithm to find an optimal position of equivalent sources with few monopoles. ${ }^{97,98}$ Pavic $^{99}$ formulated a new search algorithm, which is not a rigorous mathematical optimization procedure but a technique halfway between the latter and a simply arbitrary selection, therefore suitable for engineering applications. It looks that there is still no consensus to find out the optimal number and location of equivalent sources and they are sensitive to specific numerical algorithms. Having the position of equivalent sources as the unknown parameter and solving it along with the strength of equivalent sources as in the MFS might be an alternative way, but this will significantly increase computational cost.

Another problem with the ESM is the numerical instability. This problem is related to an ill-conditioned matrix that is influenced by the position of equivalent sources. The SVD or a regularization of the matrix ${ }^{100}$ has been a successful approach to resolve this issue. In the time domain, time averaging is also an attractive scheme ${ }^{78}$ to regularize the numerical instability. 
The time-domain ESM has been recently improved and used for wide applications. This method should be further refined to include an absorbing boundary condition and to simulate high-frequency noise for large-scale scattering surfaces.

The rigorous theories, efficient numerical algorithms, and practical applications of the ESM are continuously being developed by mathematicians and engineers. As the numerical issues are solved or carefully handled, the method will become an attractive alternative to the BEM to solve acoustic radiation and scattering problems.

\section{References}

1. S. Kobayashi and N. Nishimura, Boundary Element Methods: Fundamentals and Applications (Springer, 1992).

2. S. Sauter and C. Schwab, Boundary Element Methods, Springer Series in Computational Mathematics (Springer, 2011).

3. Q. Huang and T. A. Cruse, Some notes on singular integral techniques in boundary element analysis, Int. J. Numer. Methods Eng. 36 (1993) 2643-2659.

4. M. Carley, Numerical quadratures for singular and hypersingular integrals in boundary element methods, SIAM J. Sci. Comput. 29 (2007) 1207-1216.

5. M. Dehghan and H. Hosseinzadeh, Calculation of 2D singular and near singular integrals of boundary elements method based on the complex space C, Appl. Math. Model. 36 (2012) $546-560$.

6. J. Rong, L. Wen and J. Xiao, Efficiency improvement of the polar coordinate transformation for evaluating BEM singular integrals on curved elements, Eng. Anal. Bound. Elem. 38 (2014) 89-93.

7. M. Ochmann, The source simulation technique for acoustic radiation problems, Acoustica $\mathbf{8 1}$ (1995) 512-527.

8. V. D. Kupradze and M. A. Aleksidze, The method of functional equations for the approximate solution of certain boundary value problems, USSR Comput. Math. Math. Phys. 4 (1964) 82126.

9. R. L. Johnston and G. Fairweather, The method of fundamental solutions for problems in potential flow, Appl. Math. Model. 8 (1984) 265-270.

10. G. Karageorghis and A. Fairweather, The method of fundamental solutions for the numerical solution of the biharmonic equation, J. Comput. Phys. 69 (1987) 434-459.

11. M. A. Golberg, The method of fundamental solutions for poisson's equation, Eng. Anal. Bound. Elem. 16 (1995) 205-213.

12. D. L. Young, S. J. Jane, C. M. Fan, K. Murugesan and C. C. Tsai, The method of fundamental solutions for 2D and 3D stokes problems, J. Comput. Phys. 211 (2006) 1-8.

13. D. L. Young, C. W. Chen, C. M. Fan, K. Murugesan and C. C. Tsai, Method of fundamental solutions for stokes flows in a rectangular cavity with cylinders, Eur. J. Mech.-B/Fluids 24 (2005) 703-716.

14. D. L. Young, C. C. Tsai, K. Murugesan, C. M. Fan and C. W. Chen, Time-dependent fundamental solutions for homogeneous diffusion problems, Eng. Anal. Bound. Elem. 28 (2004) 1463-1473.

15. D. L. Young, C. C. Tsai and C. M. Fan, Direct approach to solve nonhomogeneous diffusion problems using fundamental solutions and dual reciprocity methods, J. Chin. Inst. Eng. 27 (2004) 597-609.

16. P. S. Kondapalli, D. J. Shippy and G. Fairweather, Analysis of acoustic scattering in fluids and solids by the method of fundamental solutions, J. Acoust. Soc. Am. 91 (1992) 1844-1854. 
17. A. Fairweather, G. Karageorghis and P. A. Martin, The method of fundamental solutions for scattering and radiation problems, Eng. Anal. Bound. Elem. 27 (2003) 759-769.

18. A. Bogomolny, Fundamental solutions method for elliptic boundary value problems, SIAM J. Numer. Anal. 22 (1985) 644-669.

19. M. A. Goldberg and C. S. Chen, Discrete Projection Methods for Integral Equations (Southampton Computational Mechanics Publications, 1996).

20. A. Poullikkas, A. Karageorghis and G. Georgiou, Methods of fundamental solutions for harmonic and biharmonic boundary value problems, Comput. Mech. 21 (1998) 416-423.

21. G. Fairweather and A. Karageorghis, The method of fundamental solutions forelliptic boundary value problems, Adv. Comput. Math. 9 (1998) 69-95.

22. Y. S. Smyrlis and A. Karageorghis, A linear least-squares MFS for certain elliptic problems, Numer. Algor. 35 (2004) 29-44.

23. Y. S. Smyrlis, The method of fundamental solutions: A weighted least-squares approach, BIT Numer. Math. 46 (2006) 163-194.

24. R. Kress and A. Mohsen, On the simulation source technique for exterior problems in acoustics, Math. Methods Appl. Sci. 8 (1986) 585-597.

25. A. Boag, Y. Leviatan and A. Boag, Analysis of acoustic scattering from fluid bodies using a multipoint source model, IEEE Trans. Ultrasonics, Ferroelectrics, Frequency Control 36 (1989) $119-128$.

26. A. Boag, Y. Leviatan and A. Boag, Analysis of two-dimensional acoustic scattering from periodic structures using a hybrid source model, J. Acoust. Soc. Am. 86 (1989) 387-394.

27. A. Boag, Y. Leviatan and A. Boag, Analysis of three-dimensional acoustic scattering from doubly periodic structures using a source model, J. Acoust. Soc. Am. 91 (1992) 572-580.

28. D. J. Shippy, P. S. Kondapalli and G. Fairweather, Analysis of acoustic scattering in fluids and solids by the method of fundamental solutions, Math. Comput. Model. 14 (1990) 74-79.

29. S. Ramakrishna and P. R. Stepanishen, Acoustic scattering from cylinders with a plane of symmetry using internal multipole line source distributions II, J. Acoust. Soc. Am. 93 (1993) 673-682.

30. C. J. S. Alves and S. S. Valtchev, Numerical comparison of two meshfree methods for acoustic wave scattering, Eng. Anal. Bound. Elem. 29 (2005) 371-382.

31. R. Mathon and R. L. Johnston, The approximate solution of elliptic boundary-value problems by fundamental solutions, SIAM J. Numer. Anal. 14 (1977) 638-650.

32. A. Karageorghis, B. T. Johansson and D. Lesnic, The method of fundamental solutions for the identification of a sound-soft obstacle in inverse acoustic scattering, Appl. Numer. Math. 62 (2012) 1767-1780.

33. T. Kitagawa, On the numerical stability of the method of fundamental solution applied to Dirichlet problem, Jpn. J. Ind. Appl. Math. 5 (1988) 123-133.

34. T. Kitagawa, Asymptotic stability of the fundamental solution method, J. Comput. Appl. Math. 38 (1991) 263-269.

35. A. H. Barnett and T. Betcke, Stability and convergence of the method of fundamental solutions for helmholtz problems on analytic domains, J. Comput. Phys. 227 (2008) 7003-7026.

36. T. W. Drombosky, A. L. Meyer and L. Ling, Applicability of the method of fundamental solutions, Eng. Anal. Bound. Elem. 33 (2009) 637-643.

37. P. A. Ramachandran, Method of fundamental solutions: Singular value decomposition analysis, Int. J. Numer. Methods Biomed. Eng. 18 (2002) 789-801.

38. C. S. Chen, H. A. Cho and M. A. Golberg, Some comments on the ill-conditioning of the method of fundamental solutions, Eng. Anal. Bound. Elem. 30 (2006) 405-410.

39. T. Wei, Y. C. Hon and L. Ling, Method of fundamental solutions with regularization techniques for cauchy problems of elliptic operators, Eng. Anal. Bound. Elem. 31 (2007) 373-385. 
40. J. Lin, W. Chen and F. Wang, A new investigation into regularization techniques for the method of fundamental solutions, Math. Comput. Simul. 81 (2011) 1144-1152.

41. L. Marin, Treatment of singularities in the method of fundamental solutions for two-dimensional Helmholtz-type equations, Appl. Math. Model. 34 (2010) 1615-1633.

42. C. C. Tsai, D. L. Young, C. L. Chiu and C. M. Fan, Numerical analysis of acoustic modes using the linear least squares method of fundamental solutions, J. Sound Vib. 324 (2009) 1086-1110.

43. J. Antonio, A. Tadeu and L. Godinho, A three-dimensional acoustics model using the method of fundamental solutions, Eng. Anal. Bound. Elem. 32 (2008) 525-531.

44. L. Godinho, E. Costa, A. Pereira and J. Santiago, Some observations on the behavior of the method of fundamental solutions in 3D acoustic problems, Int. J. Comput. Methods 9 (2012) 1250049.

45. E. Costa, L. Godinho, J. Santiago, C. Pereira and A. Dors, Efficient numerical models for the prediction of acoustic wave propagation in the vicinity of a wedge coastal region, Eng. Anal. Bound. Elem. 35 (2011) 855-867.

46. E. Costa, L. Godinho, J. Santiago and A. Pereira, Prediction of acoustic wave propagation in a shallow water configuration using the method of fundamental solutions, J. Comput. Acoust. 20 (2012) 1250013.

47. G. H. Koopmann, L. Song and J. B. Fahnline, A method for computing acoustic fields based on the principles of wave superposition, J. Acoust. Soc. Am. 86 (1989) 2433-2438.

48. R. D. Miller, E. T. Moyer, H. Huang and H. Überall, A comparison between the boundary element method and the wave superposition approach for the analysis of the scattered fields from rigid bodies and elastic shells, J. Acoust. Soc. Am. 89 (1991) 2185-2196.

49. G. Burgess and E. Mahajerin, A comparison of the boundary element and superposition methods, Comput. Struct. 19 (1984) 697-705.

50. R. Jeans and I. C. Mathews, The wave superposition method as a robust technique for computing acoustic fields, J. Acoust. Soc. Am. 92 (1992) 1156-1166.

51. L. Song, G. H. Koopmann and J. B. Fahnline, Numerical errors associated with the method of superposition for computing acoustic fields, J. Acoust. Soc. Am. 89 (1991) 2433-2438.

52. D. T. Wilton, I. C. Mathews and R. A. Jeans, A clarification of nonexistence problems with the superposition method, J. Acoust. Soc. Am. 94 (1993) 1676-1680.

53. A. Leblanc, R. K. Ing and A. Lavie, A wave superposition method based on monopole sources with unique solution for all wave numbers, Acta Acoust. United with Acoust. 96 (2010) 125-130.

54. J. B. Fahnline and G. H. Koopmann, A numerical solution for the general radiation problem based on the combined methods of superposition and singular-value decompostion, J. Acoust. Soc. Am. 90 (1991) 2808-2819.

55. H. B. Zhang, W. K. Jiang and C. J. Liao, The application of wave superposition algorithm to identify cyclostationary sound source, Acta Acoust. United with Acoust. 94 (2008) 649-655.

56. M. Jin, X. Zhongming, L. Shu and C. Si, Study to improve the reconstruction accuracy of wave superposition method with spherical surface, in 22 nd Int. Cong. on Sound and Vibration, Florence, Italy (2015).

57. L. Geng, X. Z. Zhang and C. X. Bi, Reconstruction of transient vibration and sound radiation of an impacted plate using time domain plane wave superposition method, J. Sound Vib. 344 (2015) 114-125.

58. M. E. Johnson, S. J. Elliott, K. H. Baek and J. Garcia-Bonito, An equivalent source technique for calculating the sound field inside an enclosure containing scattering objects, J. Acoust. Soc. Am. 104 (1998) 1221-1231.

59. L. Bouchet, T. Loyau, N. Hamzaoui and C. Boisson, Calculation of acoustic radiation using equivalent-sphere methods, J. Acoust. Soc. Am. 107 (2000) 2387-2397. 
60. R. S. J. G. Gounot and Y. Musafir, A comparative study of two variants of the equivalent sources method in scattering problems, Acta Acoust. United with Acoust. 91 (2005) 860-872.

61. M. Ochmann, The full-field equations for acoustic radiation and scattering, J. Acoust. Soc. Am. 105 (1999) 2574-2584.

62. F. Holste, An equivalent source method for calculation of the sound radiated from aircraft engines, J. Sound Vib. 203 (1997) 667-695.

63. R. Piscoya, B. Haike, M. Ochmann and P. Költzsch, Equivalent source method and boundary element method for calculating combustion noise, Acta Acoust. United with Acoust. 94 (2008) $514-527$.

64. M. H. Dunn and A. F. Tinetti, Aeroacoustic scattering via the equivalent source method, in 10th AIAA/CEAS Aeroacoustics Conf., Machester, Great Britain (2004), AIAA Paper 20042937.

65. A. F. Tinetti and M. H. Dunn, Aeroacoustic noise prediction using the fast scattering code, in 11th AIAA/CEAS Aeroacoustics Conf., Monterey, California (2005), AIAA Paper 2005-3061.

66. C. A. Reimann, A. F. Tinetti and M. H. Dunn, Engine linear optimization using the fast scattering code, in 13th AIAA/CEAS Aeroacoustics Conf., Rome, Italy (2007), AIAA Paper 2007-3494.

67. A. F. Tinetti and M. H. Dunn, Curved duct noise prediction using the fast scattering code, in 13th AIAA/CEAS Aeroacoustics Conf., Rome, Italy (2007), AIAA Paper 2007-3528.

68. M. H. Dunn and A. F. Tinetti, Application of fast multipole methods to the NASA fast scattering code, in 14th AIAA/CEAS Aeroacoustics Conf., Vancouver, British Columbia Canada (2008), AIAA Paper 2008-2875.

69. A. F. Tinetti and M. H. Dunn, Acoustic simulations of an installed tandem cylinder configuration, in 15th AIAA/CEAS Aeroacoustics Conf., Miami, Florida (2009), AIAA Paper 2009-3158.

70. A. F. Tinetti and M. H. Dunn, Scattering of high frequency duct noise by full scale hybrid wing body configurations, in 15th AIAA/CEAS Aeroacoustics Conf., Miami, Florida (2009), AIAA Paper 2009-3158.

71. A. F. Tinetti and M. H. Dunn, The fast scattering code (FSC): Validation studies and program guidelines, Tech. Rep. NASA/CR-2011-217158 (NASA, 2011).

72. A. F. Tinetti and M. H. Dunn, Scattering of simulated broadband noise by conventional and next generation aircraft, in 18th AIAA/CEAS Aeroacoustics Conf., Colorado Springs (2012), AIAA Paper 2012-2075.

73. S. Lee, J. P. Erwin and K. S. Brentner, A method to predict acoustic scattering of rotorcraft noise, J. Am. Helicopter Soc. 54 (2009) 42007.

74. M. K. Myers, On the acoustic boundary condition in the presence of flow, J. Sound Vib. 71 (1980) 429-434.

75. G. Pavić, A technique for the computation of sound radiation by vibrating bodies using multipole substitute sources, Acta Acoust. United with Acoust. 92 (2006) 112-126.

76. W. Kropp and P. U. Svensson, Application of the time domain formulation of the method of equivalent sources to radiation and scattering problems, Acoustica 81 (1995) 528-543.

77. S. Lee, K. S. Brentner and P. J. Morris, Acoustic scattering in the time domain using an equivalent source method, AIAA J. 48 (2010) 2772-2780.

78. S. Lee, K. S. Brentner and P. J. Morris, Assessment of time-domain equivalent source method for acoustic scattering, AIAA J. 49 (2011) 1897-1906.

79. S. Lee, K. S. Brentner and P. J. Morris, Time-domain approach for acoustic scattering of rotorcraft noise, J. Am. Helicopter Soc. 57 (2012) 1-12.

80. S. Lee, K. S. Brentner, F. Farassat and P. J. Morris, Analytic formulation and numerical implementation of an acoustic pressure gradient prediction, J. Sound Vib. 319 (2009) 12001221. 
81. B. Rynne, Instabilities in time-marching methods for scattering problems, Electromagnetics 6 (1986) 129-144.

82. B. Rynne and P. D. Smith, Stability of time marching algorithms for the electric field integral equation, J. Electromag. Waves Appl. 4 (1990) 1181-1205.

83. B. Rynne, Time domain scattering from arbitrary surfaces using the electric field equation, J. Electromag. Waves Appl. 5 (1991) 93-112.

84. P. J. Davies and D. B. Duncan, Averaging techniques for time-marching schemes for retarded potential integral equations, Appl. Numer. Math. 23 (1997) 291-310.

85. S. Swift, G. Blaisdell and A. Lyrintzis, An efficient time-domain equivalent source method for acoustic scattering, Int. J. Aeroacoust. 14 (2015) 133-160.

86. C. X. Bi, L. Geng and X. Z. Zhang, Cubic spline interpolation-based time-domain equivalent source method for modeling transient acosutic radiation, J. Acoust. Soc. Am. 332 (2013) 59395952.

87. X. Z. Zhang, C. X. Bi, Y. B. Zhang and L. Xu, Transient nearfield acoustic holography based on an interpolated time-domain equivalent source method, J. Acoust. Soc. Am. 130 (2011) $1430-1440$.

88. X. Z. Zhang, C. X. Bi, Y. B. Zhang and L. Xu, Sound source identification and sound radiation modeling in a moving medium using the time-domain equivalent source method, J. Acoust. Soc. Am. 137 (2015) 2678-2686.

89. S. W. Pan, W. K. Jiang and S. Xiang, Reconstruction of semi-free transient sound field under different reflection conditions using an extended interpolation time-domain equivalent source method, J. Mech. Eng. Sci. 229 (2015) 895-905.

90. S. W. Pan, W. K. Jiang, S. Xiang and X. J. Liu, An interpolated time-domain equivalent source method for modeling transient acoustic radiation over a mass-like plane based on the transient half-space green's function, Wave Motion 51 (2014) 1273-1287.

91. S. W. Pan, W. K. Jiang, H. Zhang and S. Xiang, Modeling transient sound propagation over an absorbing plane by a half-space interpolated time-domain equivalent source method, J. Acoust. Soc. Am. 136 (2014) 1273-1287.

92. I. Y. Jeon and J. G. Ih, On the holographic reconstruction of vibroacoustic fields using equivalent sources and inverse boundary element method, J. Acoust. Soc. Am. 118 (2005) 3473-3482.

93. C. X. Bi and J. S. Bolton, An equivalent source technique for recovering the free sound field in a noisy environment, J. Acoust. Soc. Am. 131 (2012) 1260-1270.

94. J. Bin, M. Y. Hussaini and S. Lee, Broadband impedance boundary conditions for the simulation of sound propagation in the time domain, J. Acoust. Soc. Am. 125 (2009) 664-675.

95. F. Q. Hu, An efficient solution of time domain boundary integral equations for acoustic scattering and its acceleration by graphics processing units, in 19th AIAA/CEAS Aeroacoustics Conf., Berlin? Germany (2013), AIAA Paper 2013-2018.

96. Y. J. R. Gounot and R. E. Musafir, On appropriate equivalent monopole sets for rigid body scattering problems, J. Acoust. Soc. Am. 122 (2007) 3195-3205.

97. Y. J. R. Gounot and R. E. Musafir, Genetic algorithms: A global search tool to find optimal equivalent source sets, J. Sound Vib. 332 (2009) 282-298.

98. Y. J. R. Gounot and R. E. Musafir, Simulation of scattered fields: Some guidelines for the equivalent source method, J. Sound Vib. 330 (2011) 3698-3709.

99. G. Pavić, An engineering technique for the computation of sound radiation by vibrating bodies using substitute sources, Acta Acoust. United with Acoust. 91 (2005) 1-16.

100. B. Lam, J. Elliott, J. Cheer and W. S. Gan, Regularisation of the equivalent source method for robust numerical modelling of acoustic scattering, in 44th Int. Cong. on Noise Control Engineering, San Francisco, CA (2015). 